\title{
Análisis Proximal, Sensorial y de Textura de Salchichas Elaboradas con Subproductos de la Industria Procesadora de Atún (Scombridae thunnus)
}

\author{
Clemente Granados*, Luis E. Guzmán y Diofanor Acevedo \\ Universidad de Cartagena, Facultad de Ingeniería, Departamento de ingeniería de Alimentos, Avenida el \\ Consulado, Calle 30 No. 48-152. Cartagena, Bolívar-Colombia (e-mail: clementecondeg@gmail.com) \\ * autor a quien debe ser dirigida la correspondencia
}

Recibido Abr. 19, 2013; Aceptado May. 29, 2013; Versión final recibida Jul. 18, 2013

\begin{abstract}
Resumen
El objetivo del presente estudio fue elaborar salchichas con los subproductos del proceso de producción de atún enlatado (carne negra, carne blanca o rayada y carne de cabeza), y evaluar sus características fisicoquímicas, sensoriales y de textura. La mejor formulación fue una que presenta características similares a las salchichas comerciales. La composición fisicoquímica se determinó según la Asociación Oficial de Química Analítica-AOAC y la aceptabilidad fue evaluada con panelistas no entrenados, utilizando una escala hedónica para sabor, color, olor y textura, obteniendo buena aceptación sensorial. Los contenidos proximales fueron: humedad, 68\%; grasa, 8,5\%; proteínas, 15,8\%; cenizas, 2,0\%; valores que cumplen con los parámetros requeridos por las Normas Técnicas Colombianas por lo que se concluye que el producto obtenido se puede considerar como una alternativa en la dieta humana.
\end{abstract}

Palabras clave: salchichas, atún, perfil de textura, subproductos.

\section{Analysis Proximal, Sensory and Texture of Elaborated Sausages By-Products from the Tuna Processing Industry (Scombridae thunnus)}

\begin{abstract}
The aim of this study was to make sausage using by-products of the production process of canned tuna (dark meat, white meat or scratched and head meat), and evaluate their physicochemical, sensory and texture characteristics. The best formulation was that having characteristics similar to commercial sausages. The physicochemical composition was determined according to the Association of Official Analytical Chemistry, AOAC and acceptability was assessed with untrained panelists using a hedonic scale for taste, color, smell and texture, obtaining good sensory acceptance. The proximal contents were: moisture, $68 \%$ fat, $8.5 \%$ protein, $15.8 \%$ ash, $2.0 \%$, values that meet the parameters required by the Colombian Technical Standards It is concluded that the product obtained could be considered as an alternative in human diet.
\end{abstract}

Keywords: sausage, tuna, texture profile, by-product. 


\section{INTRODUCCION}

El atún al ser un pez con un metabolismo muy elevado contiene una carne muy irrigada. Esta irrigación hace que su carne sea de un color oscuro característico; existiendo ciertos músculos que se utilizan más durante su actividad física. Estas zonas, necesitan una mayor irrigación y por ende poseen un color y sabor más acentuado que el resto de la carne y se la conoce como "carne negra de atún". Esta carne que representa entre 20 y $30 \%$ del peso, no es apetecida debido a su fuerte sabor, se convierte en un desecho y por ello es destinada a la elaboración de alimento para gatos o harina de pescado. En el proceso de producción de atún enlatado desde el momento de la captura hasta el despacho final se generan subproductos como son la carne de la cabeza, de la cara y residuos de las salas de proceso. Una alternativa para aumentar los rendimientos, es aprovechar éstos elaborando productos como el grated o "atún rallado" que es una mezcla de partículas reducidas a dimensiones uniformes, mediante molinos adecuados. Las excelentes propiedades del atún, y su importante volumen de captura hacen de éste una de las especies marinas de mayor potencial para el desarrollo y manufactura de una gran variedad de productos, entre ellos diversos tipos de embutidos y enlatados (Izquierdo et al. 2007; García et al.2005).

Las salchichas constituyen una de las formas más antiguas de procesar alimentos, y han sobresalido por sus características nutricionales, sensoriales y funcionales respecto de otros productos cárnicos (NTC 1325, 2008; Izquierdo et al. 2007). Diferentes investigaciones han evidenciado la potencialidad de utilizar diversos tipos de carnes en su elaboración con el fin de diversificar la presentación al consumidor, sobre todo al infantil que representa un sector importante que consume este tipo de alimentos (García et al. 2005). La salchicha de pescado es una mezcla de carnes picadas de pescado, cerdo, res o pollo, aceite vegetal, con conservantes permisibles, sal y almidón; esa mezcla es empacada en una tripa la cual es sellada y luego hervida o sometida al vapor (NTC 1325, 2008).

El estudio de la emulsión es importante, porque ésta es un sistema inestable de los cuales resulta la separación de las grasas y agua siendo éste uno de los principales problemas en la elaboración y conservación de emulsiones cárnicas, ya que se debe asegurar la estabilidad fisicoquímica del producto, y en las salchichas de cualquier especie, la formación y estabilidad de la emulsión determina su calidad (Ramos et al. 2011). En la elaboración de salchichas con carne de pescado se han presentado dificultades entre ellas, la búsqueda de la proporción adecuada de los ingredientes en la formulación, y la presencia de una textura suave indeseable que parece ser uno de los principales problemas cuando se incorporan a los productos embutidos emulsionados (Park, 1978). Esta investigación tuvo como objetivo darle un valor agregado a esta importante industria por medio del aprovechamiento de los subproductos del proceso de producción de atún enlatado para elaborar salchichas y evaluar sus características fisicoquímicas, sensoriales y de textura.

\section{MATERIALES Y MÉTODOS}

La carne negra cocida subproducto del proceso de fileteado y limpieza del atún; la carne blanca cocida o rayada y la carne roja de la cabeza del atún; utilizadas en este estudio, se adquirieron en una empresa productora de atún enlatado. La grasa de cerdo y de pollo se adquirió en un almacén de cadena, los demás ingredientes y condimentos se adquirieron en una empresa dedicada exclusivamente a la venta de insumos para la industria cárnica, todas ellas ubicadas en la ciudad de Cartagena, Colombia. Las carnes antes de ser utilizadas se lavaron en una solución de cloruro de sodio al 1\% y ácido acético para conseguir una mejor limpieza y eliminar la sangre existente, ya que ésta le imprime un sabor penetrante al producto final. Para el proceso de elaboración de las salchichas se siguió el procedimiento utilizado por Hleap y Velasco (2010).

La carne, la grasa y todos los ingredientes se colocaron en un cutter marca Mainca, modelo CM14, a excepción del hielo el cual se agregó poco a poco con el objeto de mantener la temperatura de la emulsión a temperaturas $\leq 14^{\circ} \mathrm{C}$ para evitar la desnaturalización de las proteínas. Inmediatamente después del picado, la emulsión se embute en tripa artificial calibre $22 \mathrm{~mm}$ utilizando una embutidora manual Marca Javar modelo EMV15. Las salchichas se porcionaron haciendo unidades de $\pm 12 \mathrm{~cm}$ de longitud, luego de lo cual se llevan a cocción en agua caliente a $75^{\circ} \mathrm{C}$ hasta que se alcance una temperatura interna de $71^{\circ} \mathrm{C}$, luego de lo cual son retiradas del agua caliente y se sometieron a un duchado con agua fría hasta que la temperatura interna es de $30^{\circ} \mathrm{C}$, almacenándose a $4^{\circ} \mathrm{C}$ hasta el momento de los análisis.

\section{Formulación de las salchichas}

Varias formulaciones fueron ensayadas con el fin de encontrar la proporción adecuada de los ingredientes para las salchichas, seleccionando aquella que presentara color y textura similar a las tradicionales. Las diversas formulaciones ensayadas se presentan en la Tabla 1. 


\section{Análisis proximal}

La determinación de humedad, proteína, grasa, carbohidratos y cenizas se realizó según métodos de la Asociación oficial de química Analítica (AOAC, 2003).

\section{Evaluación sensorial}

La aceptación del consumidor hacia las salchichas se evaluó basándose en las características de olor, color, sabor y textura, utilizando una escala hedónica de 4 puntos, con los siguientes descriptores: Me desagrada mucho $=1$, Me desagrada un poco $=2$, Me gusta mucho $=3$ y Me gusta muchísimo $=4$. Las muestras fueron calentadas en agua a una temperatura de $70^{\circ} \mathrm{C}$, cortadas en trozos de $1,5 \mathrm{~cm}$, identificadas con números aleatorios de tres cifras. La evaluación fue realizada en un área ventilada, de buena iluminación, libre de olores extraños, por un panel de 40 evaluadores no entrenados, a los cuales se les suministró una ficha de evaluación.

Tabla 1: Formulaciones ensayadas. ${ }^{1}$ Carne negra de atún cocida subproducto del proceso de obtención de lomos.

${ }^{2}$ Carne roja cruda obtenida de las cabezas del atún. ${ }^{3}$ Carne blanca o rayada cocida subproducto del enlatado del atún.

\begin{tabular}{|c|c|c|c|c|c|c|}
\hline Ingredientes & $\begin{array}{c}\text { Formulación } \\
1\end{array}$ & $\begin{array}{c}\text { Formulación } \\
2\end{array}$ & $\begin{array}{c}\text { Formulación } \\
3\end{array}$ & $\begin{array}{c}\text { Formulación } \\
4\end{array}$ & $\begin{array}{c}\text { Formulación } \\
5\end{array}$ & $\begin{array}{c}\text { Formulación } \\
6\end{array}$ \\
\hline $\begin{array}{l}\text { Carne negra de } \\
\text { atún cocida }^{1}\end{array}$ & 50 & 25 & & & & \\
\hline $\begin{array}{l}\text { Carne roja de la } \\
\text { cabeza cruda }^{2}\end{array}$ & & & & & 42,5 & 41,8 \\
\hline $\begin{array}{l}\text { Carne blanca de } \\
\text { atún cocida }^{3}\end{array}$ & & 25 & 50 & 46 & & \\
\hline Grasa de pollo & & & 10 & & & \\
\hline Grasa de cerdo & & & & 10,35 & 10,6 & 10,45 \\
\hline Aceite de soya & 10 & 10 & & & & \\
\hline Hielo & 15 & 15 & 15 & 15 & 11,94 & 13,05 \\
\hline Harina de trigo & 22 & 20 & 20 & & & \\
\hline Almidón de papa & & & & 5 & 5,4 & 5,22 \\
\hline Proteína de soya & & & & 20 & 26,56 & 26,12 \\
\hline Carragenina & & 1 & & & & \\
\hline Sal & 1,5 & 2 & 2 & 2 & 1,59 & 2,09 \\
\hline Azúcar & 0,5 & 0,5 & 0,3 & 0,15 & 0,11 & 0,13 \\
\hline Dióxido de titanio & & 0,5 & 0,5 & 0,4 & 0,53 & 0,26 \\
\hline Óxido de hierro & & & 0,2 & 0,1 & 0,03 & 0,047 \\
\hline Polifosfatos & & & & & 0,15 & 0,156 \\
\hline Nitrito de sodio & & & & & 0,01 & 0,057 \\
\hline Ácido ascórbico & & & & & 0,03 & 0,03 \\
\hline Pimienta & & & & & & 0,1 \\
\hline Ajo & & & & & & 0,13 \\
\hline Humo líquido & & & & & & 0,03 \\
\hline $\begin{array}{c}\text { Trisazón } \\
\text { (condimento) }\end{array}$ & & & & & 0,32 & 0,08 \\
\hline $\begin{array}{l}\text { Condimento para } \\
\text { salchicha }\end{array}$ & 1 & 1 & 2 & 1 & 0,26 & 0,26 \\
\hline
\end{tabular}

Análisis del perfil de textura (TPA)

Se utilizó un texturómetro Marca Shimadzu y se realizó la determinación del perfil de textura por medio del Rheometer software. Para esto se cortaron rodajas de salchicha de aproximadamente $1,5 \mathrm{~cm}$ de grosor y se dejaron reposar por una hora a temperatura ambiente dentro de una bolsa de polietileno para evitar la pérdida de humedad (Isaza et al. 2010). El método de TPA fue realizado mediante la aplicación de una fuerza de compresión dos veces sucesivas en las muestras, con el fin de simular la masticación humana, obteniendo la curva fuerza/tiempo y calculados los parámetros: cohesividad, dureza, gomosidad y elasticidad. Los resultados obtenidos son comparados con los reportados por Herrero (2008) y Hleap y Velasco (2010); además de los obtenidos por una salchicha comercial. 


\section{Análisis estadístico}

Para determinar diferencias estadísticas, se utilizó el programa Minitab-16 (Statistical Software, USA 2011).

\section{RESULTADOS Y DISCUSIONES}

Formulación 1. El color final del embutido fue muy oscuro, casi negro, presentando un aspecto desagradable, el producto tenía un elevado sabor a sangre y un olor no característico, de consistencia pastosa luego de la cocción y el enfriamiento, debida al empleo de harina de trigo en la formulación. La presencia de condimentos o saborizantes fue imperceptible

Formulación 2. Se realizó una nueva formulación la cual poseía tres modificaciones esenciales; en vez de utilizar solo carne negra se empleó una mezcla con carne blanca cocida rayada de atún; adicionalmente se utilizó carragenina como aglutinante para facilitar la compactación del embutido luego de la cocción y dióxido de titanio como aclarador de color. Una vez realizada la cocción y el enfriamiento los resultados obtenidos fueron los siguientes: El color del producto final había mejorado, era más claro que el anterior y de apariencia similar a las salchichas de pollo, pero persistía el sabor a sangre aunque en un nivel menor. Tampoco se pudo mejorar la consistencia pastosa y el producto tenía la apariencia de un paté. Los condimentos y saborizantes no eran todavía detectables en gran medida.

Formulación 3. Las modificaciones efectuadas sobre la formulación 2, consistieron en utilizar únicamente carne blanca cocida rayada de atún. Adicionalmente se cambió el aceite vegetal por grasa de pollo con el objeto de estimular la compactación eliminando al mismo tiempo el aglutinante; por último, se utilizó un colorante comestible como el óxido de hierro para otorgar una apariencia más natural. Una vez realizada la cocción y el enfriamiento los resultados obtenidos mostraron lo siguiente: el color de la salchicha era rosado muy atractivo a la vista, aunque persistía la consistencia pastosa, ésta al parecer originada por la utilización de harina de trigo.

Formulación 4. Debido a la consistencia pastosa, se optó por eliminar el uso de harina de trigo y en su reemplazo se recurrió a la utilización de una mezcla de proteína de soya y almidón de papa, usados regularmente en la industria de apanados. Al elaborar el producto permanecía el color rosado claro y el sabor a embutido; sin embargo, se presentaba una apariencia sintética del producto y la consistencia era compacta.

Formulación 5. Con las formulaciones anteriores el producto no alcanzaba la textura deseada, fue necesario buscar otras alternativas, se pudo establecer que la principal diferencia con las salchichas de carne de res, cerdo y pollo es que utilizan carne cruda mientras la utilizada en las pruebas estaba cocida al ingresar a la fase de mezclado. Por lo cual se efectuó una nueva revisión del proceso de obtención del enlatado de atún para detectar un punto donde se generara carne cruda. Se estableció que durante el proceso de eviscerado del pescado, las cabezas eran desechadas y enviadas a la planta de harinas. De la parte superior de las cabezas, se podían extraer pedazos considerables de carne cruda de apariencia semi-oscura y libre de sangre. Por lo que se reemplazó la carne blanca rayada y cocida por la carne cruda de las cabezas. Los ingredientes restantes de las formulaciones anteriores fueron mantenidos, cambiando la grasa de pollo por la grasa de cerdo y adicionando polifosfatos, nitrito de sodio y ácido ascórbico para dar estabilidad y facilitar el curado. El color de la salchicha fue claro y atractivo, se logró la emulsión de la pasta y la consistencia del producto era compacta, aunque el producto tenía una ligera apariencia sintética.

Formulación 6. Una vez estabilizada la pasta (Ramos et al. 2007), la fase final consistió en ajustar la fórmula. En esta etapa las pruebas consistieron en la búsqueda de un mejor sabor mediante la adición de condimentos y en mejorar la apariencia mediante un equilibrio entre el dióxido de titanio y el colorante. La salchicha de atún obtenida al final de estas pruebas cumplió con todos los requisitos organolépticos de color, olor, sabor, y consistencia.

\section{Análisis proximal}

De acuerdo a la Tabla 2, el nivel de grasa encontrado en las salchichas de la formulación No 6 es más bajo a los reportados por García y colaboradores (2005) para salchichas elaboradas con carne de res, carnero, pollo y cerdo en las que se alcanzan valores que oscilan entre 24 y $45 \%$, y de salchichas elaboradas a partir de tilapia roja en la cual se hallan valores promedios de 14,68\% (Hleap y Velasco2010). Así mismo, es menor a los límites máximos establecidos por las normas Colombianas para el contenido en grasa de salchichas elaboradas con carne de bovino (21\%) y de porcino o mezcla de bovino y porcino $(35 \%)$. 
Tabla 2: Análisis proximal de la salchicha de subproductos del atún enlatado.

\begin{tabular}{|l|l|l|l|l|}
\hline Humedad (\%) & Grasa (\%) & Proteínas (\%) & Cenizas (\%) & Carbohidratos (\%) \\
\hline $68+/-0,16$ & $8,5+/-0,35$ & $15,8+/-0,09$ & $2,0+/-0,05$ & $5,7+/-0,46$ \\
\hline
\end{tabular}

El contenido de humedad y carbohidratos es mayor que los reportados por Hleap y Velasco (2010) para salchichas de tilapia. Probablemente estos valores altos en el contenido de humedad se deban a que el atún, ingrediente cárnico utilizado en la elaboración de las salchichas, se caracteriza por una elevada capacidad de retención de agua (García et al. 2005).Sin embargo estos valores son inferiores a los reportados por Cabello y colaboradores (1995), quienes encontraron valores de 73,7\% en salchichas de pescado a base de la fauna acompañante del camarón. El contenido de proteínas es superior a los establecidos por la Norma Técnica Colombiana- NTC 1325 (2008)para salchichas de cerdo y bovino de 11 y $12 \%$ respectivamente, y a los reportados por Cabello y colaboradores (1995) para salchichas de pescado (17\%)y los hallados por García et al. (2005) de 15,55\%; en formulaciones de salchichas con atún y carne de res, mientras que el contenido de cenizas fue menor en comparación con el embutido de tilapia (2,9+/$0,05 \%)$. En general los diferentes componentes de la salchicha de atún se encontraron cerca de los valores estipulados por la NTC 1325 (2008). Por lo que se podría considerar como una alternativa viable en la dieta de los consumidores.

\section{Evaluación sensorial}

Los resultados obtenidos en la evaluación sensorial de las salchichas de atún, fueron: para sabor $(3,17)$, olor $(3,15)$, color $(3,20)$ y textura $(3,30)$. La salchicha alcanzó valores superiores en todos los atributos, encontrándose entre "me gusta muchísimo" y "me gusta mucho". Los valores más bajos asignados por los evaluadores fueron atribuidos al intenso sabor y olor a pescado. Vareltzis y colaboradores (1989) reportan resultados similares para salchichas elaboradas con pasta de merluza (Merluccius merluccius) señalando que el rechazo por parte del consumidor puede ser atribuido a la presencia de un sabor a pescado no deseado, el cual fue mucho más intenso en la medida en que el porcentaje de pescado fue incrementado. García et al. (2005), reportan mayor aceptación en salchichas elaboradas sustituyendo parcialmente la carne de res por carne de atún. Para la salchicha de la formulación №6 el color fue similar al de las salchichas comerciales.

De acuerdo con los resultados obtenidos por parte de los panelistas, las salchichas presentaron una textura suave que fue de su agrado. Resultados contrarios fueron señalados por Park et al. (1988), quienes evaluaron salchichas elaboradas remplazando parcialmente la carne de res por merluza, y encontraron que los panelistas rechazaban la textura suave, afirmaron que esta característica representa uno de los mayores problemas cuando en el proceso de elaboración de las salchichas se adicionan pulpas de productos marinos.

\section{Análisis del perfil de textura (TPA)}

Los resultados de TPA para la formulación seis se muestran en la Tabla 3. Al evaluar la textura de la salchicha de atún, se observó una dureza inferior y mayor elasticidad, cohesividad y gomosidad comparados con los valores reportados por Herrero (2008) para la salchicha de carne de res y a las reportadas para salchicha de tilapia por Hleap y Velasco (2010). Al comparar los resultados sensoriales con los de TPA, confirman la aceptación por los panelistas al ser parecidos a los de las salchichas comerciales.

Tabla 3: Análisis de textura de la salchicha de atún.

\begin{tabular}{|c|c|}
\hline Parámetro & Valor promedio por repeticiones \\
\hline Dureza [N] & $24,6 \mp 0,2$ \\
\hline Elasticidad & $0,923 \mp 0,04$ \\
\hline Cohesividad & $0,773 \mp 0,051$ \\
\hline Gomosidad [N] & $19,01 \mp 0,004$ \\
\hline
\end{tabular}




\section{CONCLUSIONES}

A partir de la carne de la cabeza del atún, se elaboraron salchichas con características fisicoquímicas y sensoriales que cumplen con los parámetros requeridos por las Normas Técnicas Colombianas .Por lo que se podría considerar como una opción viable en la dieta de los consumidores y una alternativa a la producción de harina de pescado. La carne negra de atún y el atún rayado no se consideran como materia prima para la elaboración de salchichas debido a que son carnes cocidas y no forman emulsión.

\section{REFERENCIAS}

AOAC, Association of official Methods of Analytical Chemist Inc. Washington, U.S.A., Chapter 32: 1, 2, 5 y 14 (2003).

Cabello, A.M. y otros tres autores, Nuevos productos pesqueros en la dieta del venezolano, FONAIAP Divulga 49(12), 19-23 (1995).

García, A. y otros cinco autores, Formulación de salchichas con atún y carne: vida útil y aceptabilidad, Revista científica, FCV-LUZ, 15(3), 272-278 (2005).

Herrero, A.M. y otros cinco autores, Tensile properties of cooked meat sausages and their correlation with texture profile analysis (TPA) parameters and physico-chemical characteristics, Meat Science, 80(3), 690696 (2008).

Hleap, J.I. y A.V. Velasco, Análisis de las propiedades de textura durante el almacenamiento de salchichas elaboradas a partir de Tilapia roja (Oreochromis sp.), Revista de la Facultad de Ciencias Agropecuarias, Unicauca, 8(2), 46-56 (2010).

Isaza, J. y otros cuatro autores, Producción y propiedades funcionales de plasma bovino hidratado en embutido tipo salchichón, Revista Colombiana de Ciencias Pecuarias, 23(2), 199-206 (2010).

Izquierdo, P. y otros cinco autores, Análisis proximal, microbiológico y evaluación sensorial de salchichas elaboradas a base de Cachama Negra (Colossoma macropomum), Revista Científica, FCV-LUZ, 17(3), 294300 (2007).

NTC 1325, Instituto Colombiano de Normas Técnicas y Certificación (Colombia), Norma Colombiana sobre productos cárnicos no enlatados, Bogotá (2008).

Park, E.Y., C. J. Brekke y A.L. Branen, Use of pacific hake(Merluccius products) in frankfurter formulation, J. Food Sci., 43(6), 1637-1645 (1988).

Ramos y otros tres autores, Estabilidad de salchichas con hidrocoloides y emulsificantes, Inf. Tecnol., 15(4), 91-94 (2004).

Vareltzis, $\mathrm{K}$ y otros tres autores, Use of hake (Merluccius merluccius) Surimi in frankfurter formulation, Journal of Food Sci. Tech., 24(3), 227-281 (1989). 